J. Austral. Math. Soc. Ser. B 25 (1983), 2-15

\title{
CYCLIC GRAVITY WAVES IN DEEP WATER
}

\author{
P. J. BRYANT'
}

(Received 19 July 1982; revised 13 September 1982)

\begin{abstract}
Numerical evidence is presented for the existence of unsteady periodic gravity waves of large height in deep water whose shape changes cyclically as they propagate. It is found that, for a given wavelength and maximum wave height, cyclic waves with a range of cyclic periods exist, with a steady wave of permanent shape being an extreme member of the range. The method of solution, using Fourier transforms of the nonlinear surface boundary conditions, determines the irrotational velocity field in the water and the water surface displacement as functions of space and time, from which properties of the waves are demonstrated. In particular, it is shown that cyclic waves are closer to the point of wave breaking than are steady permanent waves of the same wave height and wavelength.
\end{abstract}

\section{Introduction}

A number of surprising properties have been discovered in recent years for steady periodic gravity waves of large height. These properties, reviewed by Schwartz and Fenton [4], are associated with different forms of non-uniqueness or multivaluedness in steady waves whose height is near the maximum. Attention is directed here towards unsteady periodic gravity waves of large height, partly because most naturally occurring water waves are unsteady, and partly to study the approach to wave breaking.

This investigation began with the development of a numerical method for obtaining periodic wave group solutions of permanent envelope in which neither the small wave height nor the narrow wave-band assumptions were made. Solutions were calculated as the envelope length to wavelength ratio was decreased step by step until the two lengths were equal. It was discovered then that solutions with the two lengths equal still existed as the envelope velocity was

\footnotetext{
' Mathematics Department, University of Canterbury, Christchurch, New Zealand (C) Copyright Australian Mathematical Society 1983
} 
increased step by step. This set of periodic wave group solutions, with the envelope length equal to the wavelength, and with a range of envelope velocities, is the subject of the present investigation.

It is simpler to interpret these waves in a frame of reference moving with the wave velocity rather than with the envelope velocity. Relative to the wave velocity, the shape of the wave train changes cyclically as it propagates, as though it were a periodic wave train set into an oscillation about a permanent shape. If this interpretation is accepted, it is reasonable also to accept that there should be a range of periods of shape oscillation, dependent on the amplitude of the shape oscillation, because the phenomenon is nonlinear. The range of periods of shape oscillation in the wave interpretation is equivalent to a range of envelope velocities in the envelope interpretation. Although the numerical calculations were made relative to a frame of reference moving with the envelope velocity, they are presented here in terms of the wave interpretation.

\section{Theory}

One-dimensional inviscid irrotational periodic gravity waves of wavelength $2 \pi l$, of maximum height $a$ above the mean horizontal water surface, with an amplitude ratio $\varepsilon=a / l$, are described by

$$
\begin{gathered}
\phi_{x x}+\phi_{y y}=0 \text { for } y<\varepsilon \eta(x, t), \\
\phi_{x}, \phi_{y} \rightarrow 0 \text { as } y \rightarrow-\infty, \\
\eta_{t}-\phi_{y}+\varepsilon \eta_{x} \phi_{x}=0 \text { for } y=\varepsilon \eta(x, t), \\
\eta+\phi_{t}+\frac{1}{2} \varepsilon\left(\phi_{x}^{2}+\phi_{y}^{2}\right)=0 \text { for } y=\varepsilon \eta(x, t) .
\end{gathered}
$$

The dimensional variables are the surface displacement $a \eta$, the velocity potential $(g l)^{1 / 2} a \phi$, and $l x, l y,(l / g)^{1 / 2} t$. The two lengths scales $a, l$ have been chosen for reasons of convenience in the calculations. The true wave height (crest above trough) to wavelength ratios are stated in each of the examples presented below.

Symmetric permanent wave solutions exist for which

$$
\begin{gathered}
\eta=\sum_{k} a_{k} \cos \{k(x-c t)\}, \\
\phi=\sum_{k} b_{k} e^{k y} \sin \{k(x-c t)\} .
\end{gathered}
$$

The nonlinear algebraic equations for the first $2 N$ amplitudes $a_{k}, b_{k}, k=$ $1,2, \ldots, N$, and the velocity $c$, obtained by substituting the terminated series for $\eta$ and $\phi$ into equations (2.1c, d), may be solved numerically for a given value of $\varepsilon$ by one of a number of methods. For instance, the $2 N+1$ variables may be found by 
solving the $2 N$ boundary conditions $(2.1 \mathrm{c}, \mathrm{d})$ at each of $N$ equally spaced points over a half-wavelength $0 \leqslant x-c t \leqslant \pi$, together with the kinematic equation $\eta=1$ at $x-c t=0$. This in essence is the method used by Rienecker and Fenton [2]. Alternatively, the $N$ points may be equally spaced in the $y$-direction over the above half-wavelength, using successive approximations to achieve this spacing. A third method is to calculate Fourier transforms of the boundary conditions $(2.1 \mathrm{c}$, d) after the terminated series for $\eta$ and $\phi$ have been substituted, to find numerically $a_{k}, b_{k}, k=1,2, \ldots, N$, and $c$. This is the method used in the present investigation.

Cyclic periodic wave solutions exist for which

$$
\begin{gathered}
\eta=\sum_{j} \sum_{k} a_{j k} \cos \{k(x-c t)+j \alpha t\}, \\
\phi=\sum_{j} \sum_{k} b_{j k} e^{k y} \sin \{k(x-c t)+j \alpha t\} .
\end{gathered}
$$

The wave velocity is $c$ in the sense that, relative to a frame of reference moving with velocity $c$, the wave shape oscillates with an angular frequency $\alpha$ about a symmetric shape. The terminated series for $\eta$ and $\phi$ are substituted into the boundary conditions $(2.1 \mathrm{c}, \mathrm{d})$ and double Fourier transforms with respect to $x-c t$ and $t$ are calculated numerically to find a finite number of amplitudes $a_{j k}$, $b_{J k}$ and the frequency $\alpha$ for given values of $\varepsilon$ and $c$.

Before explaining the method in more detail, the amplitudes $a_{j k}, b_{j k}$ are discussed as functions of $j$ and $k$. Each $j$ is regarded as defining a waveband containing all harmonics with wavenumbers $k \geqslant 0$, and amplitudes $a_{j k}, b_{j k}$ for the given value of $j$. The dominant waveband $j=0$, containing amplitudes $a_{0 k}, b_{0 k}$, $k=1,2, \ldots$, describes the time independent contribution to the cyclic wave in a frame of reference moving with the wave velocity. The amplitude $a_{00}$ is zero because $\eta$ has a zero mean, and the amplitude $b_{00}$ is zero since any other value in the expansion (2.3b) is meaningless. The waveband $j=1$ contains amplitudes $a_{1 k}$, $k=1,2, \ldots$, and $b_{1 k}, k=0,1,2, \ldots$, with $a_{10}$ being equal to zero because $\eta$ has a zero mean. The waveband $j=-1$ contains amplitudes $a_{-1, k}, k=1,2, \ldots$, and $b_{-1, k}, k=1,2, \ldots$, since $b_{-1,0}$ is included with $b_{10}$. Similarly, successive wavebands $j=2,3, \ldots$, and $j=-2,-3, \ldots$, each contain amplitudes $a_{j k}, b_{j k}, k=1,2, \ldots$, together with $b_{j 0}$ when $j>0$. The trend of the magnitudes of the amplitudes is towards zero as $|j|$ and $k$ increase, with $\left|b_{j k}\right|$ tending towards zero much faster in general than $\left|a_{j k}\right|$.

The series $(2.3 \mathrm{a}, \mathrm{b})$ are substituted now into the boundary conditions $(2.1 \mathrm{c}, \mathrm{d})$ with the abbreviations

$$
\begin{gathered}
c_{j k}=\cos \{k(x-c t)+j \alpha t\}, \\
s_{j k}=\sin \{k(x-c t)+j \alpha t\} .
\end{gathered}
$$


If the resulting expressions are denoted by $F$ and $G$, then

$$
\begin{gathered}
F=\sum_{j} \sum_{k}\left\{(k c-j \alpha) a_{j k} s_{j k}-k b_{j k} e^{\varepsilon k \eta} s_{j k}\right\} \\
-\varepsilon\left(\sum_{j} \sum_{k} k a_{j k} s_{J k}\right) \times\left(\sum_{j} \sum_{k} k b_{j k} e^{\varepsilon k \eta} c_{\jmath k}\right)=0 \\
G=\sum_{j} \sum_{k}\left\{a_{j k} c_{j k}-(k c-j \alpha) b_{j k} e^{\varepsilon k \eta} c_{j k}\right\} \\
+\frac{1}{2} \varepsilon\left(\sum_{j} \sum_{k} k b_{j k} e^{\varepsilon k \eta} c_{j k}\right)^{2}+\frac{1}{2} \varepsilon\left(\sum_{j} \sum_{k} k b_{j k} e^{\varepsilon k \eta} s_{j k}\right)^{2}=0
\end{gathered}
$$

where $e^{\varepsilon k \eta}=\exp \left\{\varepsilon k \Sigma_{p} \Sigma_{q} a_{p q} c_{p q}\right\}$. The definition of $\varepsilon$ is equivalent to

$$
H=\sum_{J} \sum_{k} a_{j k}=1 .
$$

Equations $(2.4 \mathrm{a}, \mathrm{b})$ may be transformed numerically to

$$
\begin{aligned}
& F=\sum_{m} \sum_{n} F_{m n} s_{m n}=0, \\
& G=\sum_{m} \sum_{n} G_{m n} c_{m n}=0,
\end{aligned}
$$

from which

$$
F_{m n}=G_{m n}=0 \text { for all } m \text { and } n .
$$

The Fourier coefficients $F_{m n}, G_{m n}$ are nonlinear functions of $a_{j k}, b_{j k}$ and $\alpha$. Equations (2.6) may be solved numerically by Newton's method, which for $F$ is described by

$$
\begin{aligned}
\sum_{j} \sum_{k}\left(\frac{\partial F}{\partial a_{j k}}\right)_{m n}\left(a_{j k}-a_{j k}^{\prime}\right) & +\sum_{j} \sum_{k}\left(\frac{\partial F}{\partial b_{j k}}\right)_{m n}\left(b_{j k}-b_{j k}^{\prime}\right) \\
& +\left(\frac{\partial F}{\partial \alpha}\right)_{m n}\left(\alpha-\alpha^{\prime}\right)=F_{m n},
\end{aligned}
$$

for all $m$ and $n$. Each coefficient on the left of equation (2.7) is an $m, n$ Fourier coefficient of a partial derivative of equation (2.4a), and the prime denotes the new value of each variable. The coefficients and the right of equation (2.7) are evaluated at the old values of the variables. There is a similar set of equations derived from $G$ and a single equation derived from $H$. The complete set of linear equations is solved numerically for the differences $a_{j k}-a_{j k}^{\prime}, b_{j k}-b_{j k}^{\prime}, \alpha-\alpha^{\prime}$, the new values of the variables are calculated, and the process is repeated until the differences are less than some small arbitrary number $\left(10^{-10}\right.$ in the examples following). 
The range of solutions was explored as the amplitude ratio $\varepsilon$ and the wave velocity $c$ were changed step by step. In general, the method converged to the cyclic wave solutions without difficulty, the only exception being for cyclic wave solutions near to permanent wave solutions, when care had to be taken to keep the solution converging on the right path. One advantage of the Fourier transform method is that the Fourier coefficients $F_{m n}, G_{m n}$ may be found for wavebands $m$ and wavenumbers $n$ outside those included in the calculation. This information then shows which wavebands and which wavenumbers should be included in the calculation to improve the precision with which equations $(2.5 \mathrm{a}, \mathrm{b})$ are satisfied over the complete rarge of $x$ and $t$.

\section{Examples}

A range of cyclic wave solutions exists for certain given values of the amplitude ratio $\varepsilon$ as the wave velocity $c$ is changed. As $c$ was increased at given $\varepsilon$, the cyclic wave solutions tended towards permanent wave solutions with the amplitudes tending towards zero outside the $j=0$ waveband and $\alpha$ tending towards a finite limit. Each range of solutions had a lower bound as $c$ was decreased at given $\varepsilon$, but it has not been possible to isolate the physical constraint which determines this lower bound. No cyclic wave solutions could be found for $\varepsilon$ less than 0.261 , when the wave height to wavelength ratio is 0.0737 as the cyclic wave passes through its maximum height and the cyclic wave is almost a permanent wave. It appears that cyclic waves on deep water exist only at wave height to wavelength ratios which are more than one half of the maximum wave height to wavelength ratio for steady waves of permanent shape on deep water.

Although the range of solutions could be explored with equations $(2.5 \mathrm{a}, \mathrm{b})$ satisfied to low precision with few harmonics, computer restrictions meant that only a few examples could be calculated to high precision with many harmonics. Four such examples are presented below, with the second, third and fourth examples containing as many harmonics as could be manipulated in double precision by a Prime 750 computer.

The profiles of the four cyclic wave solutions over one wavelength and one cycle of shape, relative to a frame of reference moving with the wave velocity, are sketched in Figure 1. Starting from the symmetric initial shape, the crest moves backwards, then forwards through the centre, and finally back to the centre. It is noted, from equation (2.3a), that

$$
\eta(x-c t, t)=\eta(2 \pi-(x-c t), 2 \pi / \alpha-t) .
$$

The symmetric shape in the middle of the cycle $(t=\pi / \alpha)$ is flatter than that at the ends of cycle $(t=0,2 \pi / \alpha)$. The data for the examples are now summarised. 

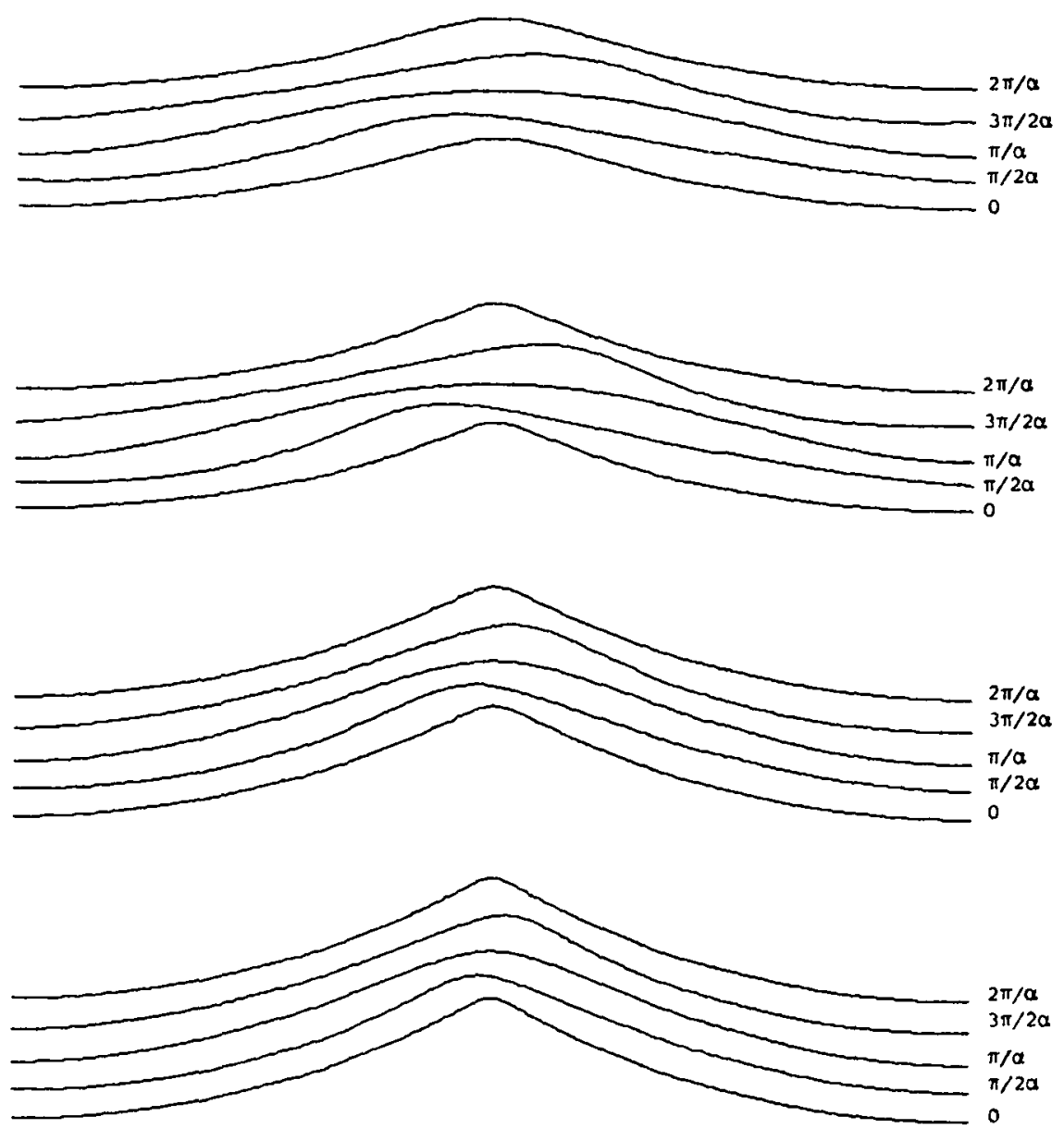

Figure 1. The profiles of each of the cyclic waves over one wavelength and one cycle of shape, relative to a frame of reference moving with the wave velocity. The times within the cycle are shown on the right of the profiles. Each profile is drawn to the same horizontal and vertical scales.

Example 1. $\varepsilon=0.3, c=1.0373, \alpha=0.5273$.

The wave height to wavelength ratio at maximum wave height in the cycle is 0.0757 with a particle velocity at the crest of 0.3749 at this instant. The final solution contains 217 harmonics (435 variables) in 14 wavebands $-3 \leqslant j \leqslant 10$, the wavenumber range being $0 \leqslant k \leqslant 25$. The maximum Fourier coefficient $F_{m n}$, $G_{m n}$ not included has magnitude $5 \times 10^{-7}$. The maximum magnitude of $F$ and $G$ over the $64 \times 64$ points in space and time used for the final calculation is $3.8 \times 10^{-5}$ with a root mean square deviation from zero of $1.8 \times 10^{-5}$. Those harmonics with amplitudes greater than $10^{-5}$ in magnitude are tabulated in the 
Appendix. (A computer listing of all harmonics calculated for this and the following examples may be obtained from the author.)

Example 2. $\varepsilon=0.4, c=1.0404, \alpha=0.4904$.

The wave height to wavelength ratio at maximum wave height in the cycle is 0.0953 with a particle velocity at the crest of 0.5637 at this instant. The final solution contains 301 harmonics (603 variables) in 17 wavebands $-3 \leqslant j \leqslant 13$, the wavenumber range being $0 \leqslant k \leqslant 31$. The maximum Fourier coefficient $F_{m n}, G_{m n}$ not included has magnitude $5.2 \times 10^{-5}$. The maximum magnitude of $F$ and $G$ over the $64 \times 64$ points in space and time used for the final calculation is $3.6 \times 10^{-3}$ with a root mean square deviation from zero of $2.6 \times 10^{-4}$.

Example 3. $\varepsilon=0.5, c=1.0672, \alpha=0.3972$.

The wave height to wavelength ratio at maximum wave height in the cycle is 0.1215 with a particle velocity at the crest of 0.7188 at this instant. The final solution contains 295 harmonics ( 591 variables) in 14 wavebands $-3 \leqslant j \leqslant 10$, the wavenumber range being $0 \leqslant k \leqslant 34$. The maximum Fourier coefficient $F_{m n}, G_{m n}$ among those not included in the final calculation has magnitude $1.0 \times 10^{-4}$. The maximum magnitude of $F$ and $G$ over the $128 \times 64$ points in space and time is $3.05 \times 10^{-2}$ with a root mean square deviation from zero of $1.5 \times 10^{-3}$. (The calculation of the final solution from the previous solution containing 39 fewer harmonics took about 24 hours of central processor time on a Prime 750 computer.)

Example 4. $\varepsilon=0.55, c=1.0799, \alpha=0.3199$.

The wave height to wavelength ratio at maximum wave height in the cycle is 0.1319 with a particle velocity at the crest of 0.8303 at this instant. The final solution contains 303 harmonics ( 607 variables) in 13 wavebands $-3 \leqslant j \leqslant 9$, the wavenumber range being $0 \leqslant k \leqslant 30$. The maximum Fourier coefficient $F_{m n}, G_{m n}$ among those not included in the final calculation has magnitude $8.2 \times 10^{-4}$. The maximum magnitude of $F$ and $G$ over the $64 \times 64$ points in space and time used for the final calculation is $5.2 \times 10^{-2}$ with a root mean square deviation from zero of $3.2 \times 10^{-3}$. The wave profiles and the properties described below were plotted both for the final solution and for the previous solution containing 28 harmonics less, when the only discernible difference was a slight sharpening of the crest in the final solution. Even though it would be preferable to include more harmonics, it does appear that the properties are accurate to within the precision with which the figures are drawn. 
It is of interest to compare each of the cyclic wave examples with a corresponding permanent wave example, in particular the permanent wave with the same wave height and wavelength as the cyclic wave has at each end of a cycle $(t=0,2 \pi)$. Each permanent wave is intermediate in shape between the symmetric profiles of the corresponding cyclic wave at the times $0, \pi / \alpha$, sketched in Figure 1. The wave velocities of the four permanent waves are 1.0287, 1.0459, $1.0751,1.0876$, each being greater than the wave velocity of the corresponding cyclic wave. The particle velocities at the crests of the four permanent waves are $0.3068,0.4198,0.6237,0.7509$, each being less than the particle velocity of the corresponding cyclic wave. The ratio of the maximum particle velocity at the wave crest to the wave velocity for the cyclic waves is $0.365,0.542,0.674,0.769$ and for the permanent waves is $0.298,0.401,0.580,0.690$ respectively. Given the non-uniform properties that have been found for permanent waves as the limiting wave is approached, any extrapolation of properties from large wave heights to the limiting wave height must be treated with caution. However, the trends in the two sets of ratios above suggest that the ratio for cyclic waves may reach one before the limiting wave is reached, after which breaking would occur as a spilling of the wave crest.

The water motion associated with cyclic waves is examined now from both the Eulerian and Lagrangian viewpoints. The horizontal velocity profile under a wave crest at the beginning of each cycle is sketched in Figure 2 for each of the four examples of cyclic waves. The mean shear across the wave crest, defined arbitrarily as $(u(\varepsilon \eta)-u(0)) / \varepsilon \eta$ is $0.396,0.634,0.713,0.840$ respectively for the four examples. In contrast, the four permanent waves have mean shears of 0.274 , $0.368,0.550,0.685$ respectively. If the mean shear is accepted as a measure of the approach to wave breaking, a comparison of the two sets of measurements suggests that the unsteady nature of cyclic waves causes them to be closer to the point of wave breaking than are steady permanent waves of the same height and wavelength.

An alternative view of the water motion is provided by the paths followed by marked fluid particles. The path of a particle which began at a wave crest at the beginning of a cycle is sketched in Figure 3 for each of the cyclic wave examples, and in Figure 4 for each of the permanent wave examples. The location of the particle at later times is marked on each path, where $2 \pi / c$ is the wave period and $2 \pi / \alpha$ is the period of one cycle of wave shape. (A second order Runge-Kutta method was used to calculate the particle paths, whose accuracy could be checked by confirming that the particle remained on the water surface.) The main difference between the pairs of particle paths is that the cyclic wave particle paths do not have any spatial periodicity, since the shape period $2 \pi / \alpha$ is not a rational multiple of the wave period $2 \pi / c$. The net horizontal distance covered by a fluid 
particle between loops on its path is less for the cyclic waves than for the permanent waves, the reason being that the wave height and hence fluid velocity of the cyclic waves is less in the middle of a cycle $(t=\pi / \alpha)$ than at the ends $(t=0,2 \pi / \alpha)$, while the height of the permanent waves remains constant. In the first two examples of cyclic waves, the interval between the first and second loops lies in the middle of a cycle, making it shorter than the interval between the second and third loops which lies at the end of a cycle.
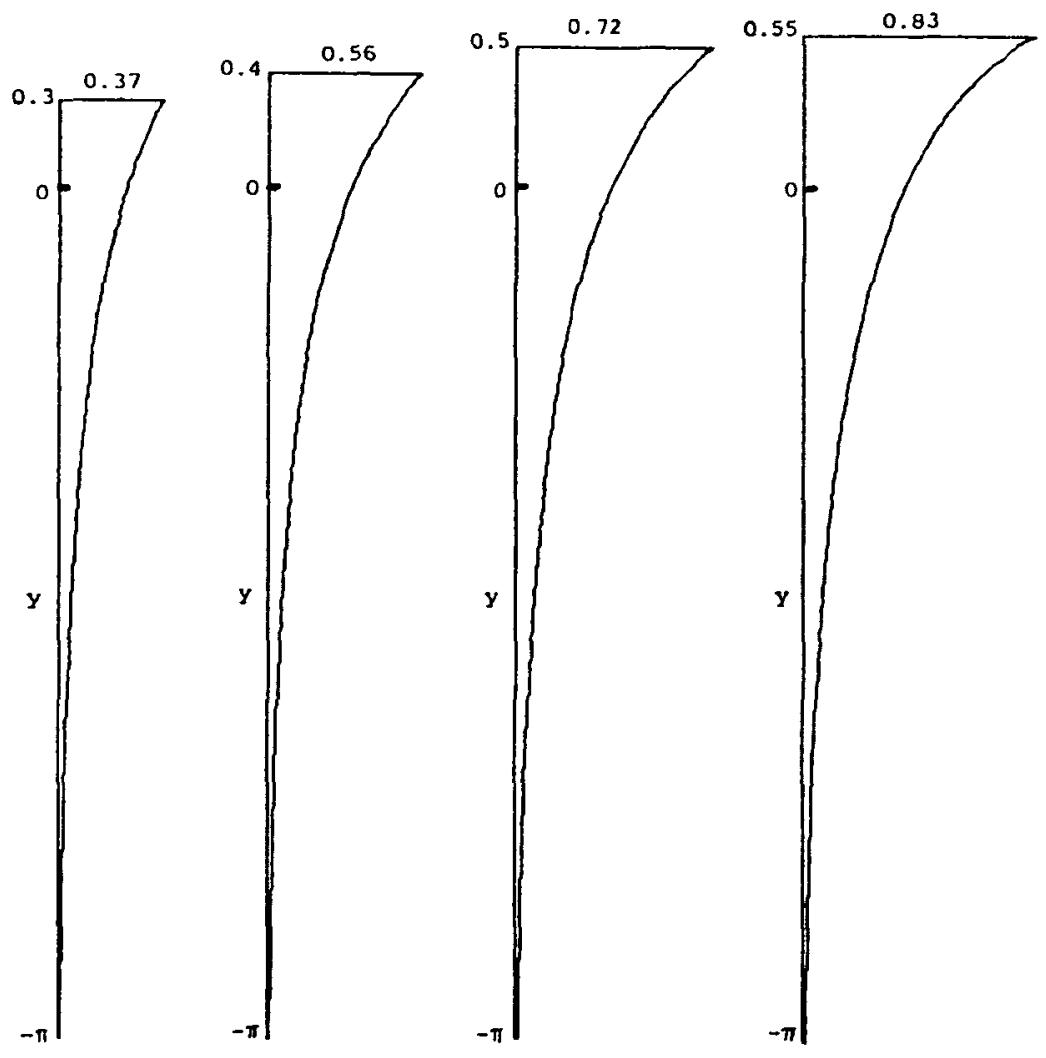

Figure 2. The horizontal velocity profile under a wave crest at the beginning of a cycle $(t=0)$, for each cyclic wave example. 


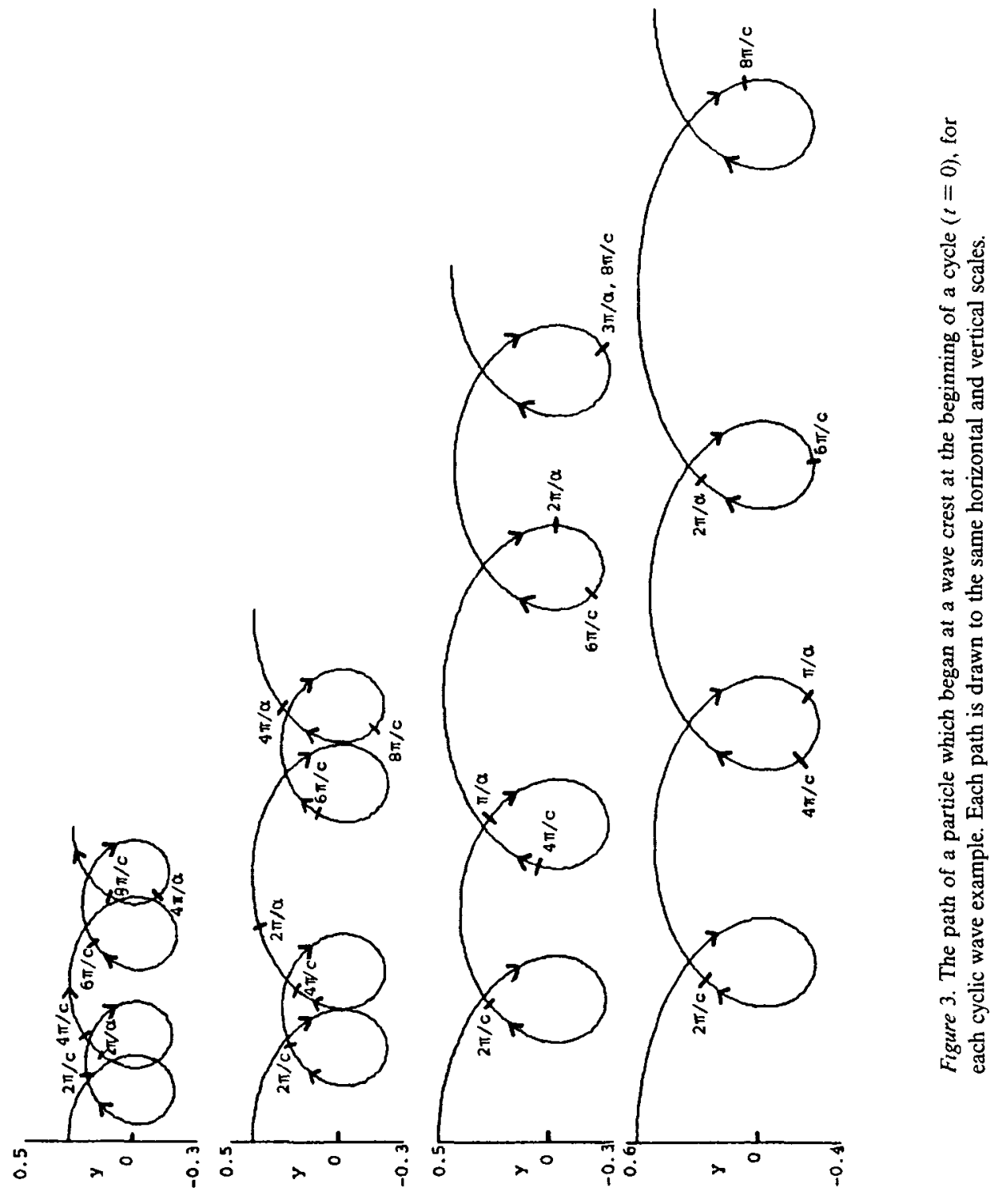




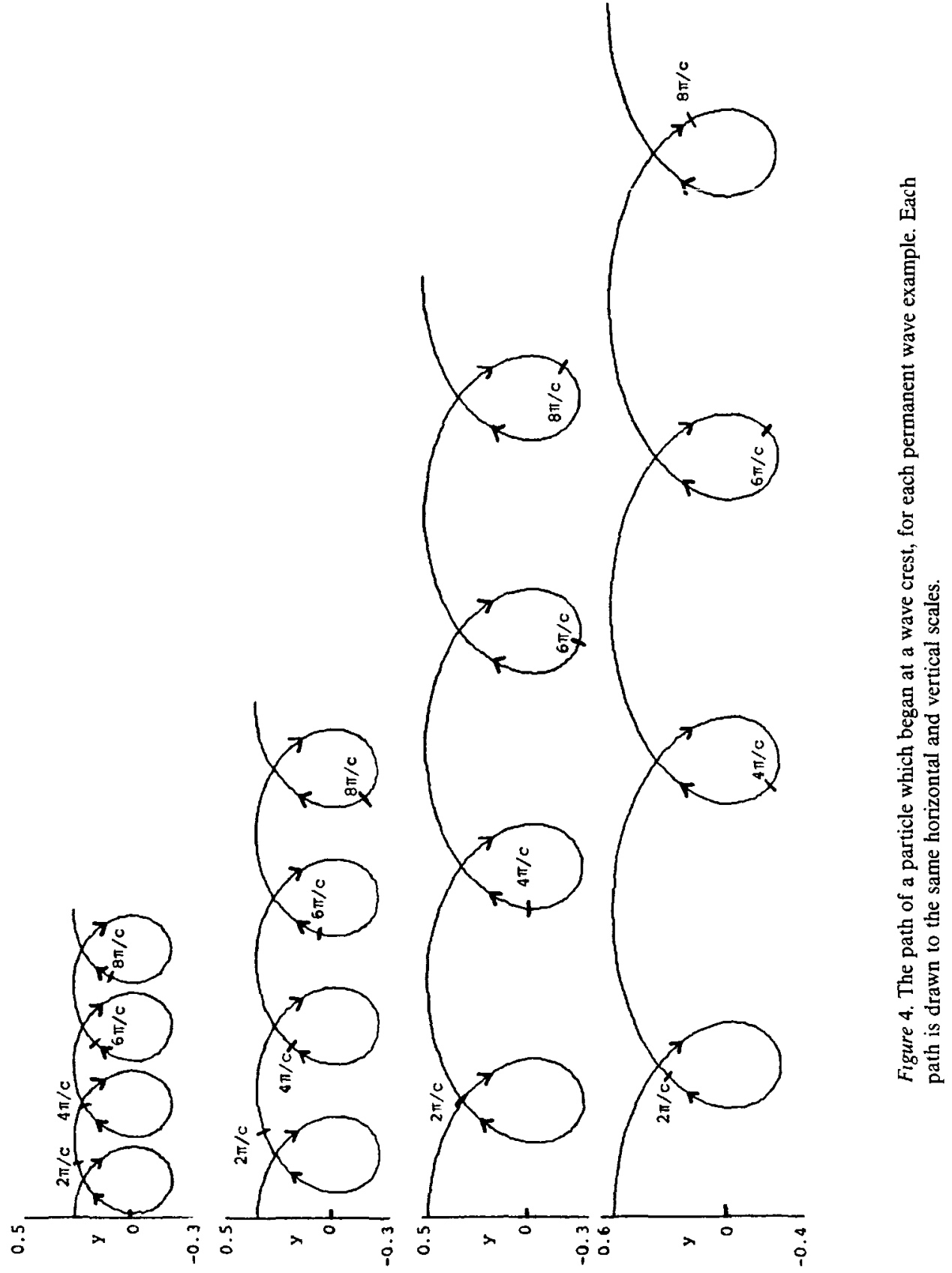




\section{Discussion}

The cyclic waves investigated here are particular unsteady solutions of the equations governing waves on deep water in irrotational invisicid two dimensional motion. The chosen form of solution (equations 2.3) is a simple unsteady generalisation of the form for single crested permanent waves (equations 2.2). It can be expected that a suitable form of solution describing a two-wavelength wave group can be found which is an unsteady generalisation of the form for double-crested permanent waves. This in turn should be capable of further generalisation to all multi-crested permanent waves of the type described by Saffman [3].

Ocean waves of large height are modelled often by steady waves of permanent form. It may be more realistic to model regular ocean waves of large height by cyclic waves, since naturally occurring waves are never completely steady. Longuet-Higgins [1] derived a number of properties of steady permanent waves near the point of wave breaking, including the existence of a sharp shear at the free surface of a steady wave of maximum steepness. The trend in the cyclic wave examples above, together with the large increase in high wavenumber harmonics in the velocity field as the wave height approaches the limiting case, suggests that a sharp shear may occur at the free surface of cyclic waves before maximum steepness is reached. Although no difficulties can be forseen in extending the range of cyclic wave solutions to larger wave heights with a larger computer, the increase in the number of high harmonics may place limits on the Fourier transform method of solution.

\section{Appendix}

Table of harmonics for Example 1 with magnitudes exceeding $10^{-5}$ :

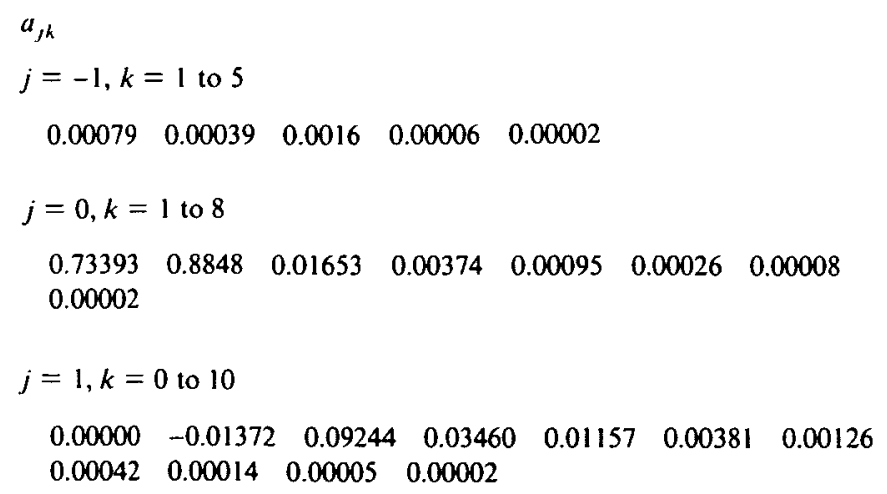




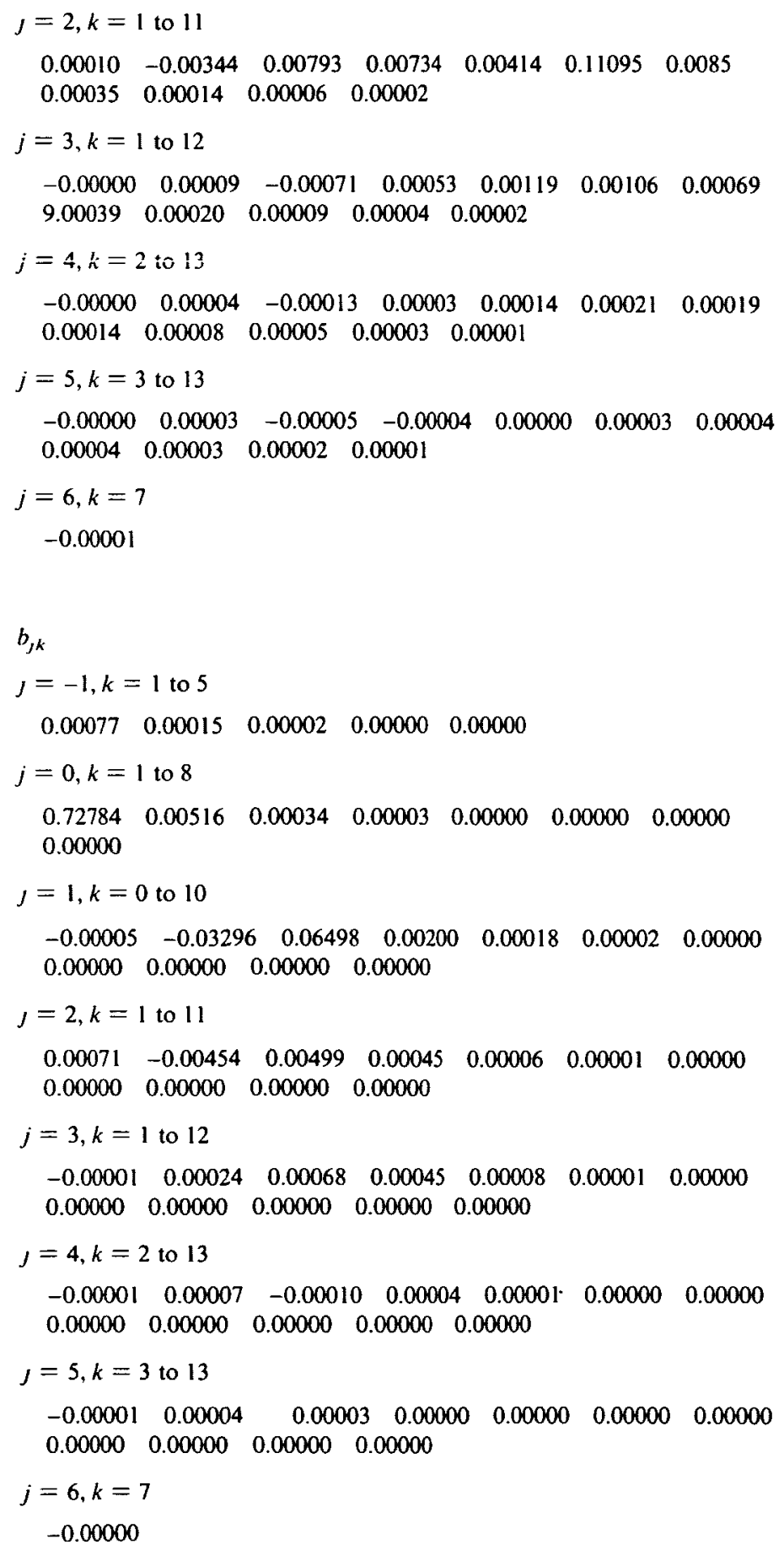




\section{References}

[I] M. S. Longuet-Higgins, "The trajectories of particles in steep, symmetric gravity waves", $J$. Fluid Mech. 94 (1979), 497-517.

[2] M. M. Rienecker and J. D. Fenton, “A Fourier approximation method for steady water waves", J. Flud Mech. 104 (1981), 119-137.

[3] P. G. Saffman, "Long wavelength bifurcation of gravity waves on deep water", J. Fluid Mech. 101 (1980), 567-581.

[4] L. W. Schwartz and J. D. Fenton, "Strongly nonlinear waves", Ann. Rev. Fluid Mech. 14 (1982), 39-60. 